Voix et Images

\title{
Deux échos québécois de grands romans épistolaires du dix-huitième siècle français
}

\section{Monique Moser-Verrey}

Volume 12, numéro 3 (36), printemps 1987

Yves Beauchemin

URI : https://id.erudit.org/iderudit/200663ar

DOI : https://doi.org/10.7202/200663ar

Aller au sommaire du numéro

Éditeur(s)

Université du Québec à Montréal

ISSN

0318-9201 (imprimé)

1705-933X (numérique)

Découvrir la revue

Citer cet article

Moser-Verrey, M. (1987). Deux échos québécois de grands romans épistolaires du dix-huitième siècle français. Voix et Images, 12(3), 512-522.

https://doi.org/10.7202/200663ar d'utilisation que vous pouvez consulter en ligne.

https://apropos.erudit.org/fr/usagers/politique-dutilisation/ 


\title{
Deux échos québécois de grands romans épistolaires du dix-huitième siècle français
}

\author{
par Monique Moser-Verrey, Université de Montréal
}

Le style épistolaire n'est pas vraiment à la mode, et cependant sa spécificité communicative tente à nouveau romanciers et essayistes. La Littérature et le reste, d'André Brochu et Gilles Marcotte offrait en 1980 une correspondance aux lecteurs. L'année dernière il y a eu les Lettres sur l'amour de Marc Chabot et Sylvie Chaput. Dans les deux cas la réflexion d'un chacun est à la fois tenue en échec et dynamisée par les apports de l'autre: jeu de hockey, disaient les premiers ${ }^{1}$, recherche commune d'intimité affirment les autres ${ }^{2}$. L'essentiel demeure en tout cas, l'échange des idées et le cheminement à deux. En tant que dialogue, l'échange de lettres s'accorde le temps d'être lent et de rester ouvert. L'œuvre de fiction, pour sa part, se plaît parfois à exploiter le potentiel des jeux intersubjectifs en mettant en scène de telles correspondances. Il se dessine alors des parcours intellectuels et émotifs, dont le lecteur doit épouser toutes les vicissitudes, comme le témoin de ce que les psychologues aiment appeler des 'bouts de chemin'.

Au dix-huitième siècle et au début du dix-neuvième siècle, la stratégie narrative du roman par lettres jouissait d'une grande popularité dans la littérature européenne. Ce type de fiction a aussi permis aux femmes de la noblesse et de la bourgeoisie montante d'entrer en littérature par le biais d'une écriture qu'elles maîtrisaient, puisque l'art de la correspondance leur était enseigné et faisait partie de leurs tâches domestiques ${ }^{3}$. Il n'est donc pas étonnant de constater que le roman épistolaire a surtout été étudié par des chercheurs qui s'intéressent soit au dix-huitième siècle, soit aux études féminines. La mode du réalisme a sans doute écarté pour un temps cette forme qui favorisait une perspective absolument subjective. Puis d'autres techniques d'écriture, comme le monologue intérieur ou encore le style indirect libre, ont permis aux romanciers d'éclairer la subjectivité de leurs personnages sans freiner le rythme de l'action, ni rompre le charme du récit par la prise de parole appuyée que représente une lettre ${ }^{4}$. Si aujourd'hui certains auteurs ne craignent plus la lourdeur des témoignages individuels, c'est peut-être que le besoin de formuler pour soi et pour l'autre les pas qui permettent à chacun de se situer dans un monde en mouvance redevient impératif. Notre société, comme celle de l'Europe du dix-huitième siècle, est en mutation. Aucun credo n'est en mesure d'offrir réponse à tout, de sorte que chacun est bien forcé de jongler avec ses propres pensées et ses propres émotions. Le genre épistolaire permet d'expliciter de telles spéculations intimes et prévoit qu'on se les approprie par une signature. C'est une forme personnalisée.

Au Québec on note la parution récente de deux œuvres de fiction épistolaires. En 1981 il y a eu Louise ou la Nouvelle Julie de Marc Gendron ${ }^{5}$ et en 1984 Lettres d'une autre de Lise Gauvin ${ }^{6}$. Cette forme n'est pas non plus 
étrangère aux anglophones. En 1982 la Noire américaine Alice Walker publiait The Color Purple ${ }^{7}$, roman épistolaire qui a gagné le prix Pulitzer et se trouve être actuellement un véritable best-seller. En 1983 le romancier canadien Clarc Blaise publiait également un très beau roman par lettres intitulé Lusts ${ }^{8}$. Ce qui distingue cependant les œuvres québécoises de ces deux romans en anglais, c'est le rapport explicite qu'elles établissent avec deux des plus fameux romans épistolaires du dix-huitième siècle français. Par son titre Marc Gendron évoque Julie ou la Nouvelle Hélö̈se de Jean-Jacques Rousseau, tandis que Lise Gauvin signe Roxane, redonnant ainsi vie au personnage féminin qui avait le dernier mot dans les Lettres persanes de Montesquieu. Ici aucun compte rendu n'a relevé ces allusions, mis à part celui d'Isabelle Ferland qui, dans le Continuum, attribue l'œuvre de Montesquieu au dix-septième siècle ${ }^{9}$. Il s'agit peut-être d'une faute d'impression, peu importe d'ailleurs. Le fait est que l'allusion littéraire porte à vide pour un public qui connaît peu la littérature européenne et encore bien moins les penseurs du dix-huitième siècle. En s'inscrivant dans une chaîne communicative qui transcende l'horizon d'attente de leur public immédiat, Gendron et Gauvin prennent un risque qui n'est certes pas gratuit. Il s'agit précisément d'ouvrir la scène locale à des mondes qui lui sont extérieurs.

Mon propos sera d'éclairer la façon dont ces deux œuvres québécoises du début des années 1980 s'inscrivent dans la tradition universelle du genre épistolaire et d'analyser le rapport qu'elles entretiennent avec les modèles romanesques auxquels elles se réfèrent. La critique distingue généralement deux tendances dans le style épistolaire, l'une didactique, l'autre sentimentale ${ }^{10}$. Il me semble que les deux textes qui nous intéressent ici illustrent chacun l'une de ces tendances. Je montrerai d'abord que les Lettres d'une autre de Lise Gauvin s'en tiennent au terrain relativement stable de la démarche didactique. Ensuite je suivrai Marc Gendron dans les eaux extrêmement troubles du parcours sentimental qu'il propose à son lecteur dans Louise ou la nouvelle Julie.

Lise Gauvin ne prétend pas avoir écrit un roman, mais plutôt un essai/ fiction. L'essai porte sur la réalité québécoise des années 1982-84. Il se divise en treize parties qui sont datées. Plusieurs remarques sur des faits de l'actualité tant politique que culturelle ancrent le texte dans l'histoire, de même que la référence à des lieux réels le fixe dans l'espace. Les deux années à partir desquelles se tisse la réflexion de l'épistolière scandent aussi deux phases distinctes de son cheminement. Dans un premier temps le lecteur découvre une sorte de balisage des contextes qui marquent la réalité québécoise. On voyage de Montréal à Québec, puis de Montréal à New York, on pénètre dans les milieux étudiants, les cercles littéraires, les familles. On se rend à Vancouver, puis on revient à Montréal pour découvrir enfin le charme estival d'une île du Saint-Laurent. C'est le calme, la retraite, la vie insulaire. Au centre du texte, il s'agit bien de la septième partie, un recentrement s'opère. L'île est appréhendée comme microcosme de la vie québécoise (p. 66) dont il faut dépasser l'apparence cauchemardesque pour en découvrir le génie. Puis la huitième partie ramène le lecteur aux questions soulevées d'entrée et concernant surtout les productions symboliques qui émanent de ce génie. La 
deuxième phase est abordée, et dès lors les thèmes de la première phase sont repris, précisés et approfondis. Les questions touchant à la langue et les questions cernant le sort des femmes réapparaissent en alternance de façon particulièrement insistante. Sans que le ton des lettres soit didactique, le rythme de leur enchaînement relève d'un excellent procédé pédagogique de présentation et de consolidation. Reste à voir si les éléments fictifs de cet ouvrage servent aussi cette volonté didactique de faire connaître tout en interrogeant.

En effet Lise Gauvin n'assume pas personnellement les lettres qui forment le corps de l'essai. Elle les attribue à une voyageuse, une immigrante d'origine persane en quête d'un pays d'adoption. Voilà où commence la fiction. La voix de l'essayiste est déplacée, pour permettre un effet de recul, une distanciation propice à l'étonnement et à la prise de conscience que tout bon pédagogue et tout bon critique doivent savoir susciter. Le stratagème du regard neuf posé sur une réalité bien connue pour mieux la saisir et mieux la juger n'a en lui-même rien de neuf. Dès lors l'évocation d'un illustre prédécesseur (p. 125) peut fonctionner comme légitimation. Mon but est de montrer comment l'allusion aux Lettres persanes travaille le texte de Lise Gauvin et l'enrichit en lui apportant plus qu'une simple légitimation. En tant que figure de style, l'allusion littéraire est tout à fait polymorphe. Elle peut aller de la citation explicite, en passant par l'évocation passagère, jusqu'au pastiche le plus secret. Dans Lettres d'une autre il faut distinguer au moins trois niveaux allusifs qui justifient et alimentent la fiction. Tout d'abord une citation de l'introduction aux Lettres persanes a été placée en exergue munie de toutes les références utiles. Ensuite l'idée de mettre un(e) voyageur(euse) d'origine orientale en présence d'une civilisation occidentale est tout simplement reprise avec quelques modifications qui ne sont pas sans intérêt. Enfin le nom de Roxane est lui-même chargé de signification. En ce qui concerne la structure de la narration la polyphonie imaginée par Montesquieu, qui fait s'entrecroiser diverses correspondances, est réduite ici au procédé monophonique du type "Werther»", où toutes les lettres émanent de la même plume et s'adressent au même correspondant. Les impressions sont recueillies sur le vif et confiées à une amie restée en Perse. La nouveauté par rapport aux Lettres persanes, c'est d'avoir affaire à une correspondance entre femmes, qui de plus se trouve être une correspondance tronquée. Il ne peut donc pas y avoir les échanges de vues informés que Montesquieu prête à ses deux voyageurs, Usbek et Rica.

En fait le rapport entre Roxane et Sarah est très ambigu. Sarah est à la fois proche et lointaine. Dans la première et dans la huitième lettre Roxane invite Sarah à venir la rejoindre, (p. 20 et 81 ) mais cette perspective est annulée par la treizième lettre où Roxane quitte (p. 119) Sarah, est sur le point de perdre son persan (p. 120) et s'apprête à se nommer Québécoise (p. 121). De plus l'intimité supposée entre ces deux femmes est quelque peu diminuée par l'aveu que fait Roxane au début de la quatrième lettre. Il s'agit là du seul élément d'intrigue qui puisse fonder la fiction de ce voyage. Roxane aurait fui son fiancé sans que son intime amie comprenne le vrai mobile de son départ. Enfin elle lui parle de la "Perse», comme si Sarah n'en vivait pas 
ÉTUDES 515

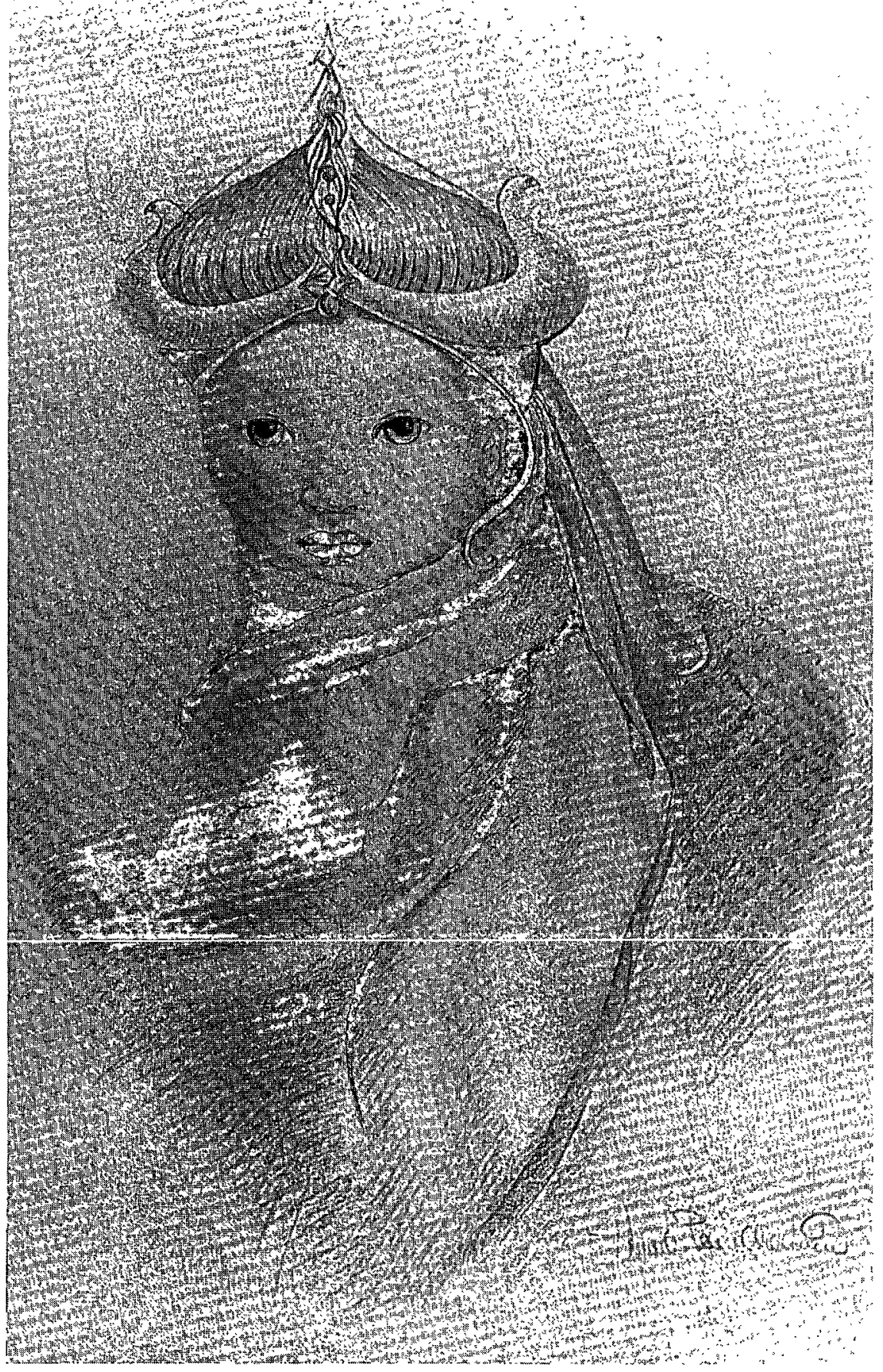


la réalité quotidienne et suppose par contre que le nationalisme et le machisme des Québécois l'inquiètent: Je ne supportais plus le nationalisme exacerbé qui commençait à poindre en notre pays. Ce monde de tradition et de pouvoir mâle exclusif. $A$ ce sujet, il me semble n'y avoir aucune commune mesure entre ce que j'ai vécu là-bas et ce que j'ai pu voir au Québec (p. 37) ${ }^{12}$.

On voit bien que le tu n'a aucune consistance et se trouve même être entièrement gommé. À vrai dire la fiction de cette relation occupe une place extrêmement mince dans le texte. Il est significatif de voir que la Perse n'apparaît d'abord que dans une parenthèse (p. 12) et que l'expérience commune des deux amies est reléguée, du point de vue grammatical, dans des propositions relatives appositives, dont la particularité est justement celle de pouvoir être omises. Voici à titre d'exemple celles qu'on peut relever dans la première lettre:

Tu sais... que dans les milieux Persans que nous fréquentions, la libération de la femme...

... Paris, où toi et moi avons fait escale un temps,...

Je découvre là tout simplement une liberté et une force auxquelles nous sommes bien loin d'arriver, dans notre pays ${ }^{13}$

Je pense que ces exemples sont suffisamment éloquents pour justifier l'idée que, tout comme le nom de Sarah, la fiction de cette relation épistolaire est simplement apposée au texte.

La question de la clôture de la correspondance s'avère enfin particulièrement révélatrice. Traditionnellement la fin d'une correspondance fictive est movitée par un événement qui prive celle-ci de sa raison d'être. Dans son étude sur l'épistolarité, Janet Gurkin fait l'inventaire des quatre possibilités classiques $^{14}$ : Le correspondant meurt, c'est le cas du Werther de Goethe. Les correspondants se réunissent, c'est le cas des personnages dans Lusts de Blaise. La correspondante renonce à sa passion, c'est le cas de la religieuse des fameuses Lettres portugaises de Guilleragues. Ou alors c'est la guérison du correspondant qui rend son écriture thérapeutique superflue, comme dans Herzog de Bellow. Ici, nous avons déjà vu, que le Je te quitte de la dernière lettre semble tout à fait gratuit. Ni le devenir Québécoise, ni la publication des lettres ne justifient cette rupture au niveau de l'intrigue. Si Roxane signe $T a$ toujours fidèle, Persane d'origine et Québécoise d'adoption (p. 125), c'est que le jeu de masques prend fin. L'origine persane de l'épistolière est au fond purement livresque, d'autant plus qu'aujourd'hui les ressortissants de ce pays se qualifient eux-mêmes d'Iraniens. Ceci dit, le nom de Roxane véhicule dans cette perspective intertextuelle un parti pris qui, mutatis mutandis, s'applique fort bien à la portée des Lettres d'une autre.

Dans les Lettres persanes le personnage de Roxane joue un rôle à la fois marginal et inquiétant. Pendant son voyage en Europe, Usbek a laissé ses 'femmes dans des sérails. Que vous êtes heureuse, Roxane, d'être dans le doux pays de Perse... (p. 58), écrit-il à celle qui nous intéresse. Puis il évoque le temps où cette femme rebelle et sauvage avait résisté à l'amour du maître en 
s'armant d'un poignard pour préserver son intégrité. Il n'y a en tout que dix lettres de femmes dans cet ouvrage qui en compte cent soixante et une. Roxane n'en signe que deux qui confirment l'une et l'autre sa volonté d'indépendance. Elle condamne la cruauté du maître, avoue l'avoir trompé, avoir su faire de son affreux sérail un lieu de délices et de plaisir. Tout en se donnant la mort elle rompt avec le langage de la soumission: Ce langage, sans doute, te paraît nouveau. Serait-il possible qu'après t'avoir accablé de douleurs, je te forçasse encore d'admirer mon courage? Mais c'en est fait :... je me meurs ${ }^{15}$.

Redonner vie à Roxane, c'est croire en quelque sorte à ce langage nouveau qui, dans un contexte moins extrême que celui du sérail, pourra s'orienter vers la vie. Roxane est un beau nom, mais il véhicule aussi une farouche volonté d'indépendance. En émigrant au Québec la nouvelle Roxane assure une relève. Une voix de femme s'affirme. Roxane va publier ses lettres et ainsi les exposer à ce que Roger Duchêne appelle la métamorphose monstrueuse qui transforme un message confidentiel en communication publique ${ }^{16}$. C'est un pas qui coûte et que Roxane n'aurait pas le courage de faire sans l'appui de Sarah. Dans ce contexte la présence de Sarah gagne enfin sa véritable raison d'être. Voici ce qu'en dit Roxane: Si l'art procède au départ d'une capacité d'entrer en communication avec soi-même, on ne saurait imaginer aucun art qui ne soit pas, au moins à un degré infime, reçu. Pour le meilleur et pour le pire, tu devras donc partager avec moi la responsabilité de ces textes! J'ai remarqué que les hommes sont souvent jaloux de cette solidarité qui lie de plus en plus les femmes entre elles (p. 97). Malgré cette solidarité sur laquelle l'auteure peut sans doute compter, l'ombre de Montesquieu la gêne et elle s'attribue une certaine modestie (p. 125) là où je parlerais plutôt d'une légitime ambition, à savoir celle d'être entendue.

Le genre épistolaire est ouvert à toutes sortes de correspondances et a toujours défié le temps, l'espace et les convenances. Essentiellement conventionnel et imitatif il offre aussi des espaces d'ancrage à de nouvelles imitations. Inscrire son propos dans un tel espace, c'est lui donner droit de cité dans ce vaste réseau de dialogues qui traversent les cultures et les siècles. Même si Lise Gauvin n'a pas trouvé ce que son personnage appelle le difficile rapport entre l'autobiographie et la fiction (p. 19), elle a su investir le langage nouveau qu'annonçait Montesquieu d'une réflexion intéressante où l'altérité, la féminité et la québécité se rejoignent.

Contrairement aux Lettres d'une autre, Louise ou la Nouvelle Julie est un véritable roman épistolaire polyphonique. Marc Gendron y met en scène Louise, une professeure de philosophie, mais au lieu d'offrir un parcours instructif, comme Lise Gauvin, il rend compte de divers cheminements sentimentaux et ses lettres, à l'instar de celles de Rousseau, reflètent l'âme de ses personnages. Cet étalage de l'intimité des êtres occulte l'intrigue, de sorte qu'il faut s'adonner à une lecture extrêmement attentive pour pouvoir reconstruire le cours des événements. Ni Madeleine Ouellette-Michalska, ni Réginald Martel n'ont pris cette peine. Leurs comptes rendus ne se basent que sur les deux premières parties de ce roman qui en compte cinq ${ }^{17}$. Pour mieux apprécier les tenants et les aboutissants de cet ouvrage très complexe, 
il est évidemment utile d'avoir présente à l'esprit la référence que Gendron affiche dans son titre. D'autre part le texte fournit lui-même certaines clefs de lecture qu'il s'agit de découvrir au milieu du carnaval des états d'âme. En fait il serait plus juste de parler d'une fête verbale, car sous le masque des mots les états d'âme des personnages s'enchaînent avec beaucoup de justesse. S'il peut y avoir une vérité qui tienne, c'est sans doute celle du parcours psychologique qui transforme la philosophe en romancière ou, si l'on préfère, le philosophe en romancier. En l'occurrence c'est la même chose et il est certain que la masculinitude (p. 205) des mâlins (p. 17) est entièrement remise en cause dans ces correspondances.

Le jeu des allusions littéraires et philosophiques est extrêmement fourni dans ce texte. Il serait tentant de soumettre tout le roman à une étude aussi poussée que celle de Ruth Amossy sur les jeux de l'allusion littéraire dans Un Beau Ténébreux de Julien Gracq ${ }^{18}$. On ne serait certes pas déçu d'apprécier les réseaux intertextuels tissés par l'allusion qui guette le lecteur à chaque page, telle l'araignée au milieu de sa toile... Hélas, mon but ne peut pas être d'étudier ici tous ces jeux. Pour rester dans le cadre de l'épistolarité qui nous intéresse ici, je me contenterai de retracer les éléments qui font appel à ce réseau. Tout en situant les cinq parties du roman, je tâcherai de montrer comment élles remettent en jeu Julie ou la Nouvelle Héloïse, mais aussi d'autres romans épistolaires du dix-huitième siècle.

Dans la première partie une étudiante nommée Clara entame avec sa professeure de philosophie, Louise, une correspondance qui conduira très vite ces deux jeunes femmes vers un amour consommé et heureux. Leur attirance mutuelle se double d'un rejet de la dominance mâle. Tandis que - Louise dénonce le discours machiste des philosophes, Clara déforme joyeusement le français pour l'adapter à ses besoins de femme. Se référant à la relation qu'elle entretient avec son frère, elle écrira par exemple: nous sommes unies l'une à l'autre et nous demeurons pourtant silencieuses l'une en face de l'autre (p. 28) ${ }^{19}$, laissant le féminin l'emporter en dépit de la grammaire. Clara se plaît aussi dans la nébulosité de ses mots. Grâce à l'écriture de son journal elle a entrevu la possibilité de détourner le langage, de se l'annexer et de l'épurer (p. 17).

Les dix-neuf lettres de cet épisode gravitent autour de la dixième qui narre la rencontre des corps et la défloration de Clara par Louise. Une rencontre des âmes par l'écriture a précédé ce moment, un hymne à l'amour le suit. Louise révèle son histoire et l'alternance régulière des lettres s'interrompt au moment où Clara emménage chez Louise. Alors que Rousseau promettait à ses lecteurs les Lettres de deux amants ${ }^{20}$, Gendron nous soumet les lettres de deux amantes. L'homosexualité fonctionne ici comme la figure d'une perversion qui se veut bien plus générale. Il s'agit de réécrire le monde en dehors des paradigmes de la société patriarcale et bourgeoise qu'exalte Rousseau et dont l'optique mâle est toujours dominante. Le bonheur de Louise et Clara, c'est de se sentir jumelles, de vibrer ensemble et de mener jusqu'au bout une intimité complice dont Rousseau a déjà peint l'image dans La Nouvelle Héloïse. Clara, c'est Claire l'inséparable amie de Julie. Dès lors on ne s'étonnera pas de la voir affubler son amie du nom tendre de l'hélouise 
des coteaux du lac (p. 50). Pour sa part Louise s'accuse d'être une nominaliste vulgaire et voudrait rebaptiser son amie Flora (p. 48). Pourquoi Flora, si ce n'est pour nier le mot défloration qui reflète évidemment la vision mâle de l'événement.

La deuxième partie du roman est entièrement consacrée à la débauche épistolaire (p. 58) qu'entretient Louise avec un autre de ses élèves. Le style de Clara était simple et direct. Par contraste ce nouveau correspondant déploie une faconde ludique, faite de mots tordus qui illustrent assez bien son esprit retors. Ayant fait le premier pas il invite Louise à lui répondre en adressant ses lettres à marc dudding, Poste restante, Bureau de poste, Outremont (p. 60). Vu le nominalisme déjà confessé, on peut supposer que ce "marc" n'est pas très étranger à l'auteur, d'autant plus que ce correspondant signera à la fin du roman sa lettre d'adieu à Louise de ce nom (p. 277). Commence alors un bal des noms d'emprunt et un grand jeu de cache-cache avec la correspondante. Même la sage Louise, qui cette fois n'oublie pas son rôle de professeure et de maîtresse à penser, finit par signer Pénélope, Xanthippe, la femme de Loth, etc. pour répondre à j.j., jean-jaque, jean-jaime, j.-jack, je-j., et j'en passe.

L'origine de ces variations sur un nom se trouve indiquée dès la première lettre: les jeunes élèves ennichés de leur profe ne m'intéressent pas unquoat... si je l'étais, j'aurais fait usage d'un stylo saint-preustien mooderne et sentimenteur, fidèle en cela à j.j. de genève en gruyère qui confond un jeune homme amourut avec un pâle et étique powète du dimanche (p. 59) ${ }^{21}$. j.j. de genève c'est bien sûr Jean-Jacques Rousseau que Louise ne manque pas non plus de citer en acceptant de se prêter à cette correspondance. Elle tient aussi à mettre en cause le style de Saint-Preux, le principal épistolier du roman de Rousseau: Le feu s'exhalait avec nos soupirs de nos lèvres brâlantes et mon coeur se mourait sous le poids de la volupté, quand tout à coup je te vis pâlir, fermer tes beaux yeux,...et tomber en défaillance. Ainsi la frayeur éteignit le plaisir et mon bonheur ne fut qu'un éclair. Rousseau ne décrit ici que le premier baiser de l'amour. Je crois bien qu'il a dû confondre ses souvenirs! (p. 63) 22 . Si l'on compare maintenant la façon dont les deux correspondants évoquent Rousseau, on s'aperçoit qu'iis s'entendent sur i'idée que son écriture est pleine de confusions dont il faut se méfier. Le discours analytique de Louise dénonce les équivoques, tandis que le style ludique du jeune homme les intensifie. En créant des mots nouveaux, il se moque de certaines options rousseauistes. Ainsi l'humeur et la modernité ne font plus qu'un dans mooderne, grâce à un détour par l'anglais mood. Le sentiment et le mensonge font bon ménage dans sentimenteur, tandis que le mot amourut relie l'amour à la mort. N'y a-t-il pas là une sorte de condensé inédit et plaisant des grandes questions qui hantent les lettres de La Nouvelle Héloïse?

Dès lors les références à La Nouvelle Hélöise se multiplient en insistant surtout sur les passages où Saint-Preux adresse ses épanchements amoureux à l'image d'une Julie absente. Celui qui signe j.j. n'est pas mieux loti que son prédécesseur et ne se cache pas de pratiquer une écriture-onanisme. Mais il réagit mal aux conseils et aux sermons de Louise. Il rêve enfin d'une plus grande intimité: Vous serez julie, je serai clair, et nous enverrons promener 
jehan-jase chez les preux et de woolmar en orbite... (p. 125). Au moment où j.j. semble prêt à sacrifier les relais littéraires qui peuplent sa saulitude et maudit les personnages de Rousseau, un nouveau modèle épistolaire s'impose. Voici comment se finit cette lettre: L'enfer ce n'est pas les autres; c'est de ne pas baiser et de vous écrire incoüto. vice-comte valdemont de merdeuil (p. 127). Les allusions abondent. Rimbaud est convoqué au passage, puis le texte se branche sur les Liaisons dangereuses de Laclos en évoquant de façon grivoise la célèbre relation épistolaire qui lie la Marquise de Merteuil au Vicomte de Valmont. À vrai dire le vice ne prend pas et La Nouvelle Héloïse reste le point de référence principal. À la fin de cette deuxième partie une troisième correspondance s'annonce entre j.j. et Eduardo, dont le nom rappelle Milord Édouard Bomston, ami de Saint-Preux. Le débat qui liera les deux gars est ici essentiellement celui qui oppose le littéraire au scientifique, mais les questions d'ordre sentimental ne sont pas écartées pour autant.

Dans la troisième partie du roman les trois correspondances s'entrecroisent. Ce mélange, encore aggravé par le travestissement systématique des salutations et des signatures, brouille les pistes pour le lecteur. Souvent ce n'est que grâce au style de la lettre que l'on peut comprendre à qui elle s'adresse. Clara est partie étudier en Europe. La séparation est douloureuse, et la perspective d'éventuels amours buissonniers (p. 181) n'a rien de réjouissant. j.j. confie toujours à Louise tout ce qui lui passe par la tête, le cour, le ventre et le cul (p. 191). Mais il signe sagement Platonix (p. 158) et intensifie par contre ses rapports avec Eduardo. Lorsque finalement il rencontre Louise, c'est pour lui dire qu'il ne voit pas la différence entre un amour réel/charnel et un amour fictif/épistolaire (p. 208). Il est très beau parleur, mais soudain il se lève et disparaît. Lui aussi s'en va en Europe poursuivre ses études. Pour sa part Louise se met à avoir des doutes sur son travail. Le phallocentrisme des philosophes l'enrage de plus en plus. Enfin elle finit par avouer sa correspondance avec j.j. à Clara dans une longue lettre qui, au grand soulagement du lecteur, résume cette aventure en termes clairs et rationnels. Là encore une référence aux plus célèbres épistoliers du dix-huitième siècle, permet de cerner le cas de j.j.: Imagine-toi saint-preux ou werther qui devrait se contenter du vocabulaire de l'anti-culture, de la soi-disant révolution sexuelle et de la pornographie pour tenter d'exorciser sa turbulence intérieure! (p. 203). L'apparition de Werther, dont les lettres ont rendu célèbre le jeune Goethe, branche le texte sur le réseau de la littérature allemande. Rien d'étonnant à cela pour le lecteur que Louise a déjà sensibilisé à ce domaine, citant ça et là de beaux poèmes en allemand. D’ailleurs j.j. écrit aussi la langue de Goethe: Nota benêt: Nur das Denken, das wir leben, hat einen Wert ${ }^{23}$ (p. 146).

Cette vérité est illustrée de façon exemplaire dans la quatrième partie du roman. L'événement qui en détermine sans doute le parcours, c'est le nouvel amour de Clara pour Silke. Louise se réfugie d'abord dans des considérations philosophiques qui déçoivent Clara, mais le mal de vivre se déclare peu à peu et les messages décousus de Louise finissent par désoler sa correspondante. Pour sa part j.j. souhaite couper tous les ponts, son style devient plus limpide de sorte que Louise gagne confiance et peut lui raconter des souvenirs d'enfance. Cette régression amène pour Louise une expérience existentielle: 
quelque chose s'est ouvert en moi. s'est brisé. sous mes yeux la matière s'annihilant. et pur regard charnel témoin de la rupture du je (p. 243). Son école lui accorde un congé et en même temps j.j. lui apprend qu'il abandonne ses études. Devenu Verterre de son propre aveu il offre à Charlotterie, nom qui rappelle la fameuse Lotte de Werther, une collection d'aphorismes. La voie de la littérature est engagée.

Dans la cinquième partie il n'y a plus que Louise et j.j. La première se livre dans une écriture nouvelle et poétique, l'autre travaille sur un roman et fait face à la page blanche. Puis il part, tandis que Louise poursuit son parcours de mort et de renaissance poétiques. Marc dudding prend définitivement congé de son idole en lui offrant un poème de Hoffmansthal et le récit d'un voyage en Orient au cours duquel le charme de la femme inaccessible a été exorcisé. Entre-temps le roman s'est fait. Tout en annonçant l'envoi du manuscrit Louise propose dans sa dernière lettre la clef de lecture suivante: Louise-Clara c'est moi, j.j. c'est l'autre, la belle affaire. / Bris-collage (p. 290). Cette révélation convoque d'une part Flaubert, mais elle rejoint aussi les options modernistes et post-modernistes des brisures et des collages. Maintenant tout se passe comme si les correspondances dont nous avons suivi les vicissitudes, illustraient les errances d'une seule âme, celle d'une jeune philosophe qui trouve le chemin de la littérature. Or Marc Gendron est philosophe de formation, comme son héroïne. Louise ou la Nouvelle Julie est son premier roman. Il est donc plausible que j.j., alias Rousseau, soit cet autre, face à qui il a pu, à l'instar de son personnage, devenir romancier. C'est ce devenir qui est en somme figuré par l'échange des lettres marquant l'évolution des diverses instances du moi. Si ce processus peut être nourri et informé par de constantes allusions à La Nouvelle Hélö̈se, c'est que l'auteur des Discours était lui aussi devenu romancier pour dire cette vaste histoire d'amour.

Pour conclure j'affirmerai avec Lise Gauvin qu'on ne peut jouer de la référence sans modifier profondément la perspective (p. 125). Comme on l'a $\mathrm{vu}$, le travail de l'allusion ne s'épuise pas dans la révélation d'analogies. Il sert surtout à modifier et réformer les discours du passé. Ainsi Lise Gauvin met en vedette un personnage tout à fait marginal de l'œuvre de Montesquieu et ce faisant elie donne une nouveile ampieur à la voix des femmes. Le même déplacement s'observe aussi dans le roman de Gendron, puisqu'il institue une femme dans le rôle du précepteur et déclasse Saint-Preux au rang des élèves. D'autre part la forme épistolaire elle-même semble servir d'autres fins dans le contexte actuel qu'elle ne le faisait dans celui du dix-huitième siècle. À l'époque, on né doutait pas de l'unicité du sujet. Il était de mise que les auteurs-s/éditeurs d'une correspondance invoquent l'authenticité des lettres offertes au public pour piquer la curiosité du lecteur. Aujourd'hui il semble que le statut fictif des correspondances soit un atout tout aussi intéressant, tant il est généralement admis que le sujet en crise se démultiplie. L'art de Gendron est d'avoir su donner une voix crédible à toutes ses potentialités contraires. Pour sa part Lise Gauvin laisse la question de l'identité en suspens, puisque son épistolière ne trouvera pas de réponse à la question de savoir comment on peut être Québécois(e) (p. 9). Dans ces deux histoires le devenir prime sur l'être, les héros sont jeunes, tournés vers l'avenir et ouverts au 
monde. Si l'on compare leurs correspondances aux lettres que François Galarneau s'écrit à lui-même, enfermé dans ses quatre murs ${ }^{24}$, on mesurera la distance qui les sépare de ce rêveur des années soixante. Les voyages de ces nouveaux héros ont lieu et leur cosmopolitisme n'a plus grand chose à envier à celui qui a marqué les lettres du dix-huitième siècle européen.

1. André Brochu / Gilles Marcotte, la Littérature et le reste, Montréal, Les Quinze, 1980 , p. 9.

2. Marc Chabot / Sylvie Chaput, Lettres sur l'amour, Montréal, Éditions Saint-Martin, 1985, p. 8. Voir aussi, plus récemment, Hugues Coriveau/Normand de Bellefeuille, $\ddot{A}$ double sens. Échanges sur quelques pratiques modernes, Montréal, Les Herbes rouges, 1986.

3. Ruth Perry, Women, Letters and the Novel, New York, AMS Press, 1980, p. 17.

4. Cette question est traitée avec perspicacité par Dorrit Cohn, la Transparence intérieure: modes de représentation de la vie psychique dans le roman, Paris, Seuil, $198 \mathrm{I}$.

5. Marc Gendron, Louise ou la nouvelle Julie, Montréal, Québec/Amérique, 1981. Pour toute référence ultérieure à ce livre, on trouvera les indications de pages insérées dans le texte.

6. Lise Gauvin, Lettres d'une autre, Montréal, l'Hexagone/Le Castor astral, 1984. Pour toute référence ultérieure à ce livre, on trouvera les indications de pages insérées dans le texte.

7. Alice Walker, The Color Purple, San Diego, Harcourt Brace Jovanovich, 1982, First Pocket Books mass-market printing December, 1985.

8. Clarc Blaise, Lusts, Markham, Ont., Penguin Books, 1984, first published by Doubleday \& Co., 1983.

9. Isabelle Ferland, "Étrangère en son propre pays", in Continuum 1985.01.28, p. 18.

10. Laurent Versini, Le roman épistolaire, Paris, PUF, 1979, p. 10, mais aussi Janet Gurkin Altman, Epistolarity, Approches to a Form, Ohio State, University Press, Columbus 1982, p. 196.

11. Cette catégorie est proposée par François Jost, “l'Évolution d'un genre: Le roman épistolaire dans les lettres occidentales", in Essais de Littérature comparée, Tome II, (Europaeana, première série), Fribourg, Suisse, Éditions Universitaires, 1968, p. 130 ss.

12. souligné par moi.

13. Les propositions relatives appositives sont soulignées par moi.

14. Gurkin Altman, op. cit., p. 154.

15. Charles-Louis de Montesquieu, Lettres persanes, Paris, Garnier Flammarion, 1964, p. 253.

16. Roger Duchêne, Écrire au temps de Mme de Sévigné: Lettres et texte littéraire, Paris, Vrin, 1982, p. 8.

17. Réginald Martel, «Des histoires de femmes et quelles histoires», in La Presse, 1981. 05.16 , p. C3 et Madeleine Ouellette-Michalska, "Quand les archétypes éclatent", in Le Devoir, 1981. 08.01. p. 12 (Culture et société).

18. Ruth Amossy, Les jeux de l'allusion littéraire dans 'Un Beau Ténébreux' de Julien Gracq, Neuchâtel, La Baconnière, 1980.

19. souligné par moi.

20. Jean-Jacques Rousseau, Julie ou la nouvelle Héloïse, Paris, édition de René Pomeau, Garnier Frères, 1960, p. 5.

21. souligné par moi.

22. souligné par moi.

23. Seule la pensée vécue est valable.

24. Jacques Godbout, Salut Galarneau, Paris, Seuil, 1967, p. 131. 